\title{
Active-Site Properties of Phrixotrix Railroad Worm Green and Red Bioluminescence-Eliciting Luciferases
}

\author{
V.R. Viviani ${ }^{1,2}$, F.G.C. Arnoldi ${ }^{2}$, B. Venkatesh ${ }^{3}$, A.J.S. Neto ${ }^{2}$, F.G.T. Ogawa ${ }^{2}$, \\ A.T.L. Oehlmeyer ${ }^{2}$ and Y. Ohmiya ${ }^{3}$ \\ ${ }^{1}$ Universidade Federal de Sao Carlos, Campus de Sorocaba, Sorocaba, SP, Brazil; ${ }^{2}$ Departamento de Biologia \\ Celular e Molecular, Instituto de Biociências, Universidade Estadual Paulista (UNESP), Av. 24 A, 1515, \\ Bela Vista, Rio Claro, SP, Brazil 13506-900; and ${ }^{3}$ Cell Dynamics Research Group, National Institute of \\ Advanced Science and Technology (AIST), 1-8-31 Midorigaoka, Ikeda, Osaka
}

Received June 19, 2006; accepted July 11, 2006

\begin{abstract}
The luciferases of the railroad worm Phrixotrix (Coleoptera: Phengodidae) are the only beetle luciferases that naturally produce true red bioluminescence. Previously, we cloned the green- (PxGR) and red-emitting (PxRE) luciferases of railroad worms Phrixotrix viviani and $P$. hirtus [OLE1]. These luciferases were expressed and purified, and their active-site properties were determined. The red-emitting PxRE luciferase displays flash-like kinetics, whereas PxGR luciferase displays slow-type kinetics. The substrate affinities and catalytic efficiency of PxRE luciferase are also higher than those of PxGR luciferase. Fluorescence studies with 8-anilino-1-naphthalene sulfonic acid and 6-p-toluidino-2-naphthalene sulfonic acid showed that the PxRE luciferase luciferinbinding site is more polar than that of PxGR luciferase, and it is sensitive to guanidine. Mutagenesis and modelling studies suggest that several invariant residues in the putative luciferin-binding site of PxRE luciferase cannot interact with excited oxyluciferin. These results suggest that one portion of the luciferin-binding site of the redemitting luciferase is tighter than that of PxGR luciferase, whereas the other portion could be more open and polar.
\end{abstract}

Key words: bioluminescence, luciferases, Phrixotrix, railroad worms.

Abbreviations: ANS, 8-anilino-1-naphthalene sulfonic acid; TNS, 6-p-toluidino-2-naphthalene sulfonic acid; DTT, dithiothreitol; PxGR, Phrixotrix viviani green-light-emitting luciferase cDNA; PxRE, Phrixotrix hirtus red-light-emitting luciferase cDNA; CoA, coenzyme A.

Beetle luciferases catalyse the ATP-activated oxidation of firefly D-luciferin, producing bioluminescence, usually in the green-yellow region. They are the most sensitive and versatile reporter genes for bioanalytical purposes (1). Most studies have focused on firefly luciferases, many of which have been cloned and sequenced (2-11). However, the physicochemical properties of only a few of them have been studied in detail $(10,11)$. The three-dimensional structure of Photinus pyralis firefly luciferase was resolved in the absence of substrates (12), and two active-site models have been proposed $(13,14)$. According to these models, the luciferin-binding site consists of residues R218, 244HHGF247, 315GG316, R330, T343, S347, A348, and I351. Although the active site of firefly luciferase was originally thought to be hydrophobic (15), more recent results suggest that it is amphiphilic (16). Click beetle luciferases, which produce different colours, have also been cloned $(17,18)$. Mutagenesis studies identified the residues important for the bioluminescence activity and colours of these luciferases (19-24). Three mechanisms

*To whom correspondence should be addressed at: Departamento de Biologia Celular e Molecular, Instituto de Biociências, Universidade Estadual Paulista (UNESP), Av. 24 A, 1515, Bela Vista, Rio Claro, SP, Brazil 13506-900. Tel: +55 19 35264149, Fax: +55 19 35236502, E-mail: viviani@rc.unesp.br have been proposed to explain bioluminescence colour modulation by beetle luciferases: (I) non-specific solvent and orientation polarizability effects $(15,16)$; (II) specific active-site interactions with the emitter (25); and (III) the effect of the conformation of the active site on the rotational properties of the oxyluciferin thiazine rings (26). Recent theoretical and experimental studies suggested that the polarization of the phenolate and enolate groups of excited oxyluciferin under the influence of active site residues determine of the emission spectra $(27,28)$. However, the contribution of each mechanism to the determination of the bioluminescence colours produced by different beetle luciferases is unclear.

The luciferases of the Phrixotrix railroad worms are the only [OLE2] luciferases that naturally catalyse bioluminescence in the red region of the spectrum. For this reason, they constitute useful models with which to study the relationship between the structures and bioluminescence colours of beetle luciferases. Because they are the only luciferases to produce red light, they have strong potential applications in a wide variety of bioanalytical contexts. Previously, we cloned the green-emitting luciferase from $P$. viviani and the red-emitting luciferase from $P$. hirtus railroad worms (29), which are $71 \%$ identical at the primary structure level. We used mutagenesis and chimerization studies based on a comparison of their primary structures to identify the structural determinants of the 
bioluminescence colours (30-33). However, despite the identification of important regions and a few residues that influence bioluminescence colours, the structural determinants and mechanisms by which these enzymes produce such different colours remain unknown. Recently, Venkatesh et al. showed that railroad worm luciferases display strong and distinct affinities for GroEL chaperones $(34,35)$. These luciferases are beginning to be used as reporter genes in mammalian cells $(36,37)$ and have wide potential applications in bioanalytical assays of pigmented samples, such as blood. Detailed physicochemical characterization of the active sites of these enzymes could help us understand how they produce different bioluminescence colours. Here, we report the expression, purification, and characterization of these luciferases. Their distinct kinetic, dynamic, and active-site properties could explain their different emission spectra.

\section{MATERIALS AND METHODS}

Reagents-D-Luciferin and $P$. pyralis luciferase were obtained from Promega (Madison, WI) and Wako Pure Chemicals (Osaka, Japan); ATP, coenzyme A, and 8-anilino-1-naphthalene-sulfonic acid (ANS) were obtained from Sigma (St Louis, MO, USA); 6- $p$-toluidino-2naphthalene sulfonic acid (TNS) was obtained from Acros (NJ, USA); nickel-agarose resin was obtained from Qiagen (Valencia, CA, USA); restriction enzymes were obtained from Takara Shuzo (Kyoto, Japan); and Complete Protease Inhibitor Cocktail Tablets were obtained from Roche (Penzberg, Germany). Polyclonal antibodies directed against firefly luciferase and Phrixotrix luciferases were raised in immunized rabbits.

Constructs-To construct N-terminal histidine-tagged luciferase expression vectors, the pCA24N vector, which contains the LacI promoter, was digested with StuI, then treated with CIAP. The primers were then phosphorylated. Both the Phrixotrix red-emitting (PxRE) and green-emitting (PxGR) luciferase cDNAs were amplified with PCR using KOD DNA polymerase, and then ligated into the StuI-digested CIAP-treated pCA24N vector. The resultant plasmids were designated pCA24N-RE and pCA24N-GR, respectively. The railroad worm Phrixotrix luciferase chimeras PxREGRRE (fragment comprising residues 1-219 and 345-546 of PxRE luciferase and residues 220-344 of PxGR luciferase) and PxRE220GR (fragment comprising residues 1-219 of PxRE luciferase and residues above 220 of PxGR luciferase) were constructed as previously described (33).

Site-Directed Mutagenesis-Site-directed mutagenesis was performed using a Stratagene mutagenesis kit (catalogue \#200518). The plasmids containing the luciferase cDNAs were amplified using Pfu Turbo DNA Polymerase and two complementary primers containing the desired mutation, using a thermal cycler (one cycle at $95^{\circ} \mathrm{C}$; and 12 cycles of $95^{\circ} \mathrm{C}$ for $30 \mathrm{~s}, 55^{\circ} \mathrm{C}$ for $1 \mathrm{~min}$, and $68^{\circ} \mathrm{C}$ for $12 \mathrm{~min})$. After amplification, mutated plasmids containing staggered nicks were generated. The products were treated with $D p n I$ to digest the non-mutated parental plasmids, and then used to directly transform Escherichia coli XL1-Blue cells. The following primers and the corresponding reverse complements were used: GR T340A (5'GGATATGGATTAGCTGAAACTTGCTGC-3'); RE H242A
(5'-GCACCGTTCCATGCTGCCTTTGGAC-3'); GR H242A (5'-GTACCGTTCCATGCTGCCTTTGGAATG-3').

Sequencing-The mutants were sequenced with the dideoxy chain termination method using a dye-labelled terminator kit specifically developed for the ABI PRISM 377 automatic sequencer (Perkin Elmer, Forster City, CA, USA). The following primers were designed to sequence the mutants: PxRE, 5'-CCATCTATGGTAATCGTATTGCTCC-3'; PxGR, 5'-CAAGTTTCAGTTAATCCATAT-3'.

Expression and Purification-One-litre cultures of $E$. coli Bl21-DE3 carrying the plasmid pCA24N-RE or pCA24N-GR were grown at $37^{\circ} \mathrm{C}$ in $\mathrm{LB}$ medium in the presence of $100 \mu \mathrm{g} / \mathrm{ml}$ of chloramphenicol until $\mathrm{OD}_{600}=0.5$. They were then induced with $0.5 \mathrm{mM}$ IPTG overnight at $18-21^{\circ} \mathrm{C}$. The cells were harvested by centrifugation at $2,500 \times g$ for $20 \mathrm{~min}$, and then resuspended in extraction buffer A $(50 \mathrm{mM}$ sodium phosphate buffer, $300 \mathrm{mM} \mathrm{NaCl}[\mathrm{pH} 7.5]$ ) in the presence of Complete Protease Inhibitor Cocktail. The cells were disrupted using a French press or alternatively by freezing/thawing on dry ice three times, followed by weak ultrasound treatment. The samples were then centrifuged again at $15,000 \times g$ for $30 \mathrm{~min}$. The supernatant was mixed with pre-equilibrated nickel-agarose resin (Qiagen) and then poured onto a mini column. The resin was washed once with buffer B (buffer A supplemented with $10 \mathrm{mM}$ ATP, $0.8 \%$ Triton $\mathrm{X}-100,20 \mathrm{mM} \mathrm{MgCl}$, $50 \mathrm{mM} \mathrm{KCl}$ ), and once with buffer $\mathrm{C}$ (buffer A supplemented with $20 \mathrm{mM}$ imidazole). The luciferases were then eluted with increments of 100-500 mM imidazole. The freshly eluted samples were dialysed against 1 liter of $50 \mathrm{mM}$ phosphate buffer containing $1 \mathrm{mM}$ ethylenediaminetetraacetic acid (EDTA), $2 \mathrm{mM}$ dithiothreitol (DTT), and $10 \%$ glycerol ( $\mathrm{pH} 7.5$ ). Ammonium sulfate precipitation was performed at $55 \%$ and $70 \%$ saturation.

Measurement of Luminescence Intensities-Bioluminescence intensities were measured with a TD3000III luminometer (Japan) with a 1P28 photomultiplier or with an ATTO model AB2200R luminometer (Tokyo, Japan). Initial maximum intensity values were used as a measure of luciferase activity and are reported in millivolts. The assays were performed by mixing $5 \mu \mathrm{l}$ of $40 \mathrm{mM}$ ATP/80 $\mathrm{mM} \mathrm{MgSO}_{4}$ with a solution consisting of $10 \mu \mathrm{l}$ of crude extract/luciferase and $85 \mu \mathrm{l}$ of $0.5 \mathrm{mM}$ luciferin in $0.1 \mathrm{M}$ Tris-HCl ( $\mathrm{pH}$ 8.0). Absolute luminescence intensities were measured in the TD3000III luminometer and were corrected based on the spectral sensitivity of the photomultiplier at the peaks of the bioluminescence spectra by multiplying the intensity of the red-emitting luciferase by forty and that of the green-emitting luciferase by ten, considering a quantum efficiency of $2.5 \%$ at $620 \mathrm{~nm}$ and $10 \%$ at $550 \mathrm{~nm}$. Absolute intensities were calculated by comparison with a radioactive light standard, a gift of the J.W. Hastings Laboratory (38).

Kinetics Measurements and KM Determination-To estimate luciferin $K_{\mathrm{M}}, 5 \mu \mathrm{l}$ of $40 \mathrm{mM}$ ATP/80 mM $\mathrm{MgSO}_{4}$ was mixed with a solution consisting of $10 \mu \mathrm{l}$ of luciferase and $85 \mu \mathrm{l}$ of $0.1 \mathrm{M}$ Tris- $\mathrm{HCl}(\mathrm{pH}$ 8.0) containing luciferin at final concentrations between 0.01 and $1 \mathrm{mM}$. Each assay was performed in triplicate. The $K_{\mathrm{M}}$ values were calculated from Lineweaver-Burk plots using peak initial intensity values $\left(I_{0}\right)$ as a measure of $V_{0}$. The relative catalytic constant $\left(k_{\text {cat }}\right)$, defined as the number of 
substrate molecules converted by a single enzyme molecule per unit time, was calculated by the ratio of $V_{\text {max }}$ values (in photons per second) to the number of luciferase molecules, using the purified fractions with highest specific activities (fraction E4). The catalytic constant of Photinus pyralis luciferase was calculated as described using a $14.9 \mathrm{mg} / \mathrm{ml}$ commercial preparation (PROMEGA). The relative catalytic efficiency of the purified enzymes was defined as the ratio of the catalytic constant to the substrate $K_{\mathrm{M}}, k_{\text {cat }} / K_{\mathrm{M}}$. The decay rate of luminescence $\left(k_{\mathrm{D}}\right)$ was calculated according to the equation $k_{\mathrm{D}}=\ln \left(I / I_{0}\right) / \Delta t$.

Fluorescence Spectra-Bioluminescence and fluorescence spectra were measured using Spex model Fluorolog and Hitachi model F4500 spectrofluorometers. Bioluminescence spectra in the presence of guanidine sulfate were obtained by mixing $50 \mu \mathrm{l}$ of purified luciferase to $450 \mu \mathrm{l}$ of a solution consisting of $0.5 \mathrm{mM}$ D-luciferin, $2 \mathrm{mM}$ ATP, $4 \mathrm{mM} \mathrm{MgSO}_{4}$ and varying concentration of guanidine sulfate $(10-200 \mathrm{mM})$ in $0.1 \mathrm{M}$ Tris-HCl buffer. To obtain the fluorescence spectrum of tryptophan from purified Phrixotrix luciferases, $50 \mu \mathrm{l}$ of purified luciferase ( $50 \mathrm{ng} / \mu \mathrm{l}$ ) was mixed with $990 \mu \mathrm{l}$ of $0.1 \mathrm{M}$ sodium phosphate buffer ( $\mathrm{pH} 7.5$ ). Fluorescence was excited at $280 \mathrm{~nm}$ and the emission spectrum was scanned at 300-450 $\mathrm{nm}$. Purified railroad worm luciferases were titrated with 1,8-ANS and 2,6-TNS by mixing $1 \mu \mathrm{M}$ fluorescent probe with $5-10 \mu \mathrm{M}$ purified enzyme in $999 \mu \mathrm{l}$ of filtered $50 \mathrm{mM}$ phosphate buffer ( $\mathrm{pH}$ 7.5). A standard curve of energy maxima as a function of the solvent polarity index (shown in parentheses) was constructed using the following solvents: formamide (7.3), methanol (6.6), dimethylsulfoxide (DMSO) (6.5), dimethylformamide (6.4), ethanol (5.2), isopropanol (4.3), ethyl acetate (4.3), and ethylic ether (2.9). Fluorescence spectra for 2,6-TNS and 1,8-ANS were obtained with excitation at $320 \mathrm{~nm}$ and scanning at 400-600 nm. The fluorescence spectra of buffers and solvents were used as blanks. The fluorescence spectra were corrected for the spectral sensitivity of the equipment.

Western Blotting-Purified samples of Phrixotrix luciferases were subjected to sodium dodecyl sulfatepolyacrylamide gel electrophoresis (SDS-PAGE) and western blotting. The denatured samples were then applied to SDS gels and subsequently electroblotted onto nitrocellulose membranes. The membranes were first incubated with primary polyclonal antibodies directed against PxRE railroad worm luciferase or histidine-tag, then with horseradish-peroxidase-labelled anti-rabbit secondary antibody, and visualized by chemiluminescence using an ECL western blotting kit (Amersham, Buckinghamshire, UK), according to the manufacturer's instructions.

Homology-Based Modelling Studies-Homology-based models of Phrixotrix railroad worm luciferases were constructed by satisfaction of spatial restraints using the three-dimensional structure of Luciola cruciata luciferase complexed with oxyluciferin and AMP as template and the Modeller 8v2 program. The models were visualized with PyMOL Molecular Graphics System version 0.97.

\section{RESULTS AND DISCUSSION}

Expression and Purification-E. coli containing the appropriate plasmids were cultured at $18-20^{\circ} \mathrm{C}$, then induced with IPTG and harvested by centrifugation.
Two extraction methods were used. In the first, the cells were disrupted using a French press; in the second, the cells were disrupted by freezing and thawing the cell suspension three times on dry ice. With the freeze/thaw method, we obtained $0.5-1 \mathrm{mg}$ of Phrixotrix luciferases per litre of culture (Fig. 1), whereas using the French press, the yield was more than $3 \mathrm{mg} /$ liter. Fractions E3 and E4 displayed the highest specific activities and were used for assays. The fraction E4 was dialysed overnight as described in section 2 , or precipitated with ammonium sulfate at $55 \%$ and $70 \%$ saturation. Most luciferase precipitated at $70 \%$ saturation.

Luciferase Activity, Kinetic and Folding PropertiesThe red-emitting luciferase displays flash-like kinetics, with fast decay followed by a slow increase and decay of intensity (Fig. 2), whereas the green-emitting luciferase usually displays slower kinetics, with slow increase and decay rates. However, we found that both the kinetics and activities of PxGR luciferase changed after incubation.

After purification, PxGR luciferase underwent gradual activation when incubated on ice, at room temperature and even at $37^{\circ} \mathrm{C}$. Upon incubation at $4^{\circ} \mathrm{C}$ in phosphate buffer ( $\mathrm{pH} 7.5)$ in the presence of $2 \mathrm{mM}$ DTT and $1 \mathrm{mM}$ EDTA, there was a 3-6-fold increase in the activity of PxGR luciferase. After dialysis, the luminescence kinetics of PxGR luciferase also changed from the slow type discussed above (Fig. 2) to the flash type, similar to those of PxRE luciferase. A similar effect was observed with the freshly resuspended ammonium-sulfate-precipitated fraction, which displayed a very slow build up of luminescence intensity in Tris buffer at $\mathrm{pH}$ 8. However, the same samples displayed flash-like kinetics after incubation at $4^{\circ} \mathrm{C}$ for two days. Since imidazole had not inhibitory effect on the activity of the dialysed samples, the observed activation could be related to a gradual refolding of the freshly purified enzyme.
A. PxRE

123

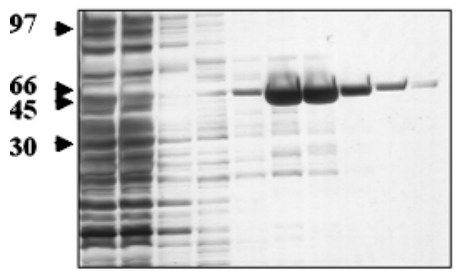

$\begin{array}{lll}1 & 2 & 3\end{array}$

\section{B. PxGR}

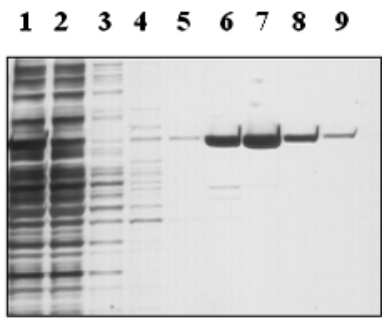

$456 \quad 6 \quad 7$

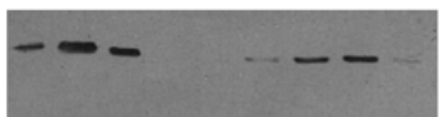

Fig. 1. His-tag affinity purification profiles for (A) PxRE and (B) PXGR luciferases. Upper panel. SDS-PAGE: lane 1, cell suspension; lane 2, cell suspension after binding to nickel-agarose resin; lanes 3-5, buffer washing; lanes $6-10$, fractions collected with increasing concentrations of imidazole (100-500 mM). Lower panel. Western blot probed with polyclonal anti-His-tag antibody: lanes 1-3, PxRE luciferase fractions collected with increasing concentrations of imidazole (200-400 mM); lanes 1-4, PxGR luciferase fractions collected with increasing concentrations of imidazole $(200-500 \mathrm{mM})$. 
Previous studies have shown that PxGR and PxRE luciferases display 25 times and 32 times higher affinities for the chaperonin GroEL, respectively, than that of firefly luciferase. This chaperone assists the refolding of these enzymes. The higher affinity of PxRE luciferase for GroEL has been attributed to its higher proportion of hydrophobic residues in the regions encompassing residues 10-30 and 100-200 (34, 35). However, we identified a large patch of hydrophobic residues exposed at the surface of PxGR luciferase, which is partially absent from PxRE luciferase that could be involved in the misfolding and aggregation of PxGR luciferase.

CoA Effect-CoA, a well-known stimulator of beetle luciferases (10), also increases the luminescence intensity of Phrixotrix luciferases. However, it had stronger effect on PxGR luciferase than on PxRE luciferase (Fig. 2). The effect of CoA deserves close attention because it is a vestigial substrate that apparently no longer plays a direct role in bioluminescence. According to an original proposal,

A

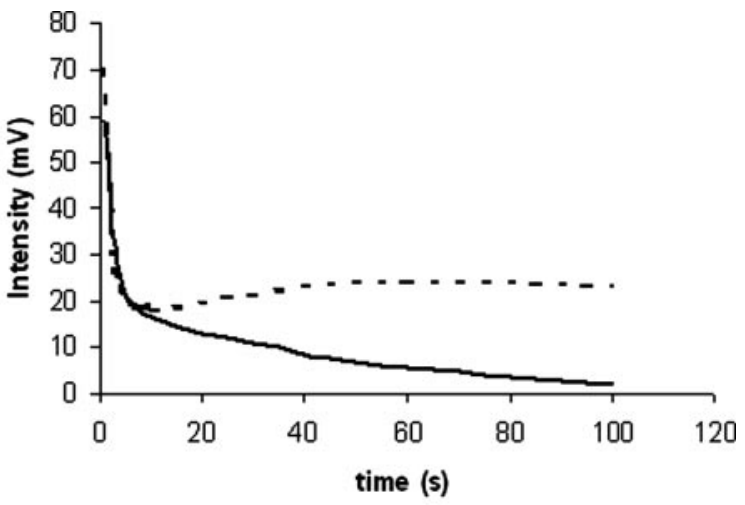

B

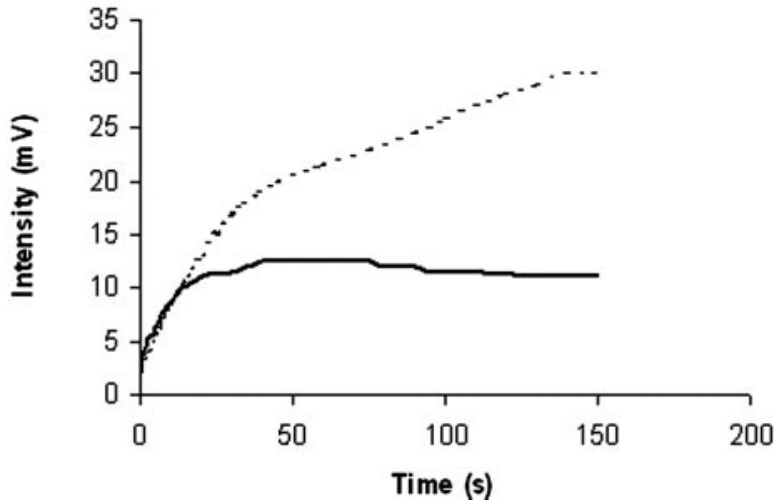

Fig. 2. Kinetic behaviour of railroad worm Phrixotrix luciferases, (A) PxRE luciferase and (B) PxGR luciferase. Full line, no addition; and dashed line, $1 \mathrm{mM}$ CoA added. the stimulating effect of $\mathrm{CoA}$ on firefly luciferase bioluminescence is attributable to the removal of the potent competitive inhibitor and autooxidation product, dehydroluciferyl adenylate, from the active site by thioesterification (10). Although firefly luciferase can adenylate both D- and L-luciferin (10), Nakamura et al. recently showed that the L-luciferin adenylate inhibitor can be removed from the active site by thioesterification with CoA, whereas D-luciferin adenylate preferentially undergoes bioluminescent oxidation (39). Studies of the threedimensional structures of homologous AMP/CoA ligases also show that CoA is involved in important conformational changes during the second reaction in these enzymes, i.e., thioesterification in most ligases and oxygenation in beetle luciferases $(40,41)$. It is remarkable that the removal of the C-terminal domain of firefly luciferase results in an impaired enzyme that produces weak red bioluminescence, indicating that the C-terminal is important for efficient yellow-green bioluminescence (42). The stronger effect of CoA on PxGR luciferase is consistent with the ability of $\mathrm{CoA}$ to promote the rotation of the $\mathrm{C}$-terminus from a closed to an open conformation in the yellow-green emitting luciferases, a step that could be partially absent in the red-emitting luciferase.

Substrate Affinities and Catalytic Efficiency-The $K_{\mathrm{M}}$ values for luciferin (Table 1) were lower than previously reported for the crude enzymes (29). Significantly, the value for PxRE luciferase is one of the lowest among the beetle luciferases (Table 1). The $K_{\mathrm{M}}$ value of PxGR luciferase for ATP is identical to the value previously reported for crude luciferase. However, the value for PxRE is considerably lower than previously reported (Table 1). In both cases, the values for ATP are similar to the values reported for other beetle luciferases. These results indicate that PxRE luciferase has higher affinities for both substrates than those of PxGR luciferase. Furthermore, although the catalytic constants were identical for both luciferases, we estimate that the relative catalytic efficiencies $\left(V_{\mathrm{M}} / K_{\mathrm{M}}\right)$ for ATP and luciferin of PxRE luciferase are at least 3 times higher than those of PxGR luciferase (Table 1), indicating that the PxRE enzyme is also catalytically more efficient. This higher catalytic efficiency could be an adaptation of the red-emitting PxRE luciferase to emit more photons in the red region in order to produce a visible functional bioluminescent signal, considering the lower visual sensitivity of most organisms to light in the red region. It is noteworthy that both Phrixotrix luciferases showed catalytic efficiency considerably lower than that of $P$. pyralis luciferase (Table 1).

Active-Site Polarity-Previously, using fluorescent probes and the luciferin competitive inhibitors 1,5-ANS and 2,6-TNS, DeLuca showed that the luciferin-binding

Table 1. Summary of physical-chemical properties of purified Phrixotrix railroadworm luciferases.

\begin{tabular}{|c|c|c|c|c|c|c|c|c|c|}
\hline \multirow{2}{*}{ Luciferase } & \multirow{2}{*}{$\begin{array}{c}\text { BL } \lambda \max ^{*} \\
\text { [Bandwidth] } \\
(\mathrm{nm})\end{array}$} & \multirow{2}{*}{$\underset{(\mathrm{kDa})}{\mathrm{MW}^{\dagger}}$} & \multirow{2}{*}{$\mathrm{pH}$} & \multicolumn{2}{|c|}{ Luciferin } & \multicolumn{2}{|c|}{ ATP } & \multirow{2}{*}{$\begin{array}{c}k_{\text {cat }} \\
\left(10^{-3} \mathrm{~s}^{-1}\right)\end{array}$} & \multirow{2}{*}{$\begin{array}{l}k \mathrm{D} \\
\left(\mathrm{s}^{-1}\right)\end{array}$} \\
\hline & & & & $\begin{array}{l}K_{\mathrm{M}} \\
(\mu \mathrm{M})\end{array}$ & $\begin{array}{l}k_{\text {cat }} / K_{\mathrm{M}^{\ddagger}} \\
\left(\mathrm{s}^{-1} \mathrm{M}^{-1}\right)\end{array}$ & $\begin{array}{l}K_{\mathrm{M}} \\
(\mu \mathrm{M})\end{array}$ & $\begin{array}{l}k_{\text {cat }} / K_{\mathrm{M}^{\ddagger}} \\
\left(\mathrm{s}^{-1} \mathrm{M}^{-1}\right)\end{array}$ & & \\
\hline P.pyralis & $562[78]$ & 60.7 & 7.9 & 10 & 3,000 & 250 & 120 & 30 & 0.024 \\
\hline PxGR & $546[70]$ & 61 & 8.0 & $64 \pm 10$ & 30 & 330 & 6 & 2 & 0.0003 \\
\hline PxRE & 623 [55] & 59.7 & 8.1 & $7 \pm 0.7$ & 285 & 130 & 15 & 2 & 0.0200 \\
\hline
\end{tabular}

*Bioluminescence spectrum maximum wavelength. $†$ Molecular weight. $\ddagger$ Catalytic efficiency. 


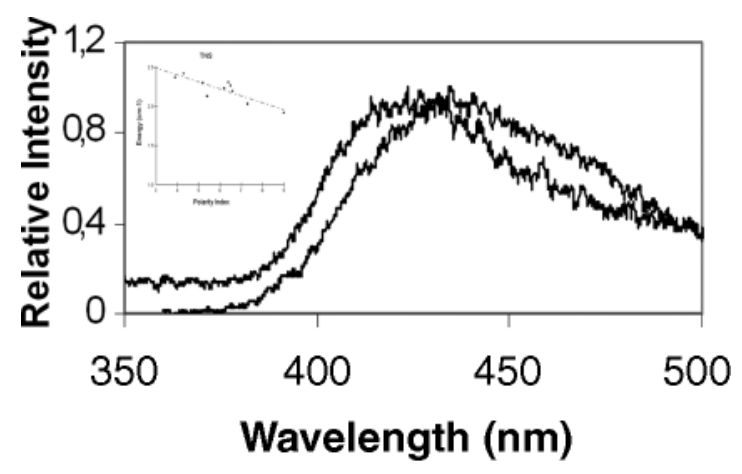

Fig. 3. Fluorescence spectra of $\mathbf{1} \mu \mathbf{M}$ 2,6-toluidinonaphthalene sulfonate in the presence of PxGR or PxRE luciferases. (Upper panel) Effect of the solvent polarity index on the energy at the peak wavelength of the fluorescence spectrum; (lower panel) Fluorescence spectra of (right) PxRE luciferase and (left) PxGR luciferase. The assays were performed in $50 \mathrm{mM}$ phosphate buffer $\mathrm{pH} 7.5$.

Table 2. Fluorescence properties of Phrixotrix railroadworm luciferases tryptophan and enzyme titrated dies.

\begin{tabular}{lccc}
\hline \multirow{2}{*}{ Luciferase } & \multicolumn{3}{c}{ Fluorescence $\lambda_{\max }(\mathrm{nm})$} \\
\cline { 2 - 4 } & Tryptophan & 2,6 TNS & 1,5 ANS \\
\hline Photinus pyralis & 334 & 428 & 471 \\
PxGR & 339 & 422 & 462 \\
PxRE & 339 & 436 & 479 \\
BSA & - & 433 & 487 \\
$1 \%$ Triton X-100 & - & 442 & - \\
$\mathrm{H}_{2} \mathrm{O}$ & - & 535 & 536 \\
\hline
\end{tabular}

site in firefly luciferase is hydrophobic (15). More recently, however, Ugarova and Brovko suggested that the active site could be amphiphilic (16). Therefore, to probe the polarity of the luciferin-binding site of the railroad-worm Phrixotrix luciferases, we measured the fluorescence spectra of the 1,8-ANS-titrated and 2,6-TNS-titrated enzymes (Fig. 3; Table 2) and compared these with the values estimated for different solvents and for P. pyralis firefly luciferase. The peaks of the TNS and ANS fluorescence spectra for PxGR luciferase were slightly blue-shifted relative to those of firefly luciferase (Table 2), whereas the peaks for PxRE luciferase were red-shifted (Table 2), indicating a more polar active site. Furthermore, in addition to the main peak, the fluorescence spectrum of TNS in PxRE luciferase (Fig. 3; Table 2) also displayed a red-shifted shoulder, which may indicate partial exposure of the active site to the solvent. Noteworthy, bovine serum albumin and Triton X-100 solutions, which enhance luciferyl-adenylate red chemiluminescence (43), display TNS fluorescence spectra quite similar to PxRE luciferase, suggesting that the binding site and environment experienced by TNS in BSA and micelles are amphiphylic and similar to the luciferin binding site nof PxRE luciferase (Table 2).

Although the effect of the solvent dielectric constant on the position of the fluorescence spectrum peak does not always display a simple linear relationship because of the complexity of the effects, it can provide a first estimate of the polarity of the luciferin-binding site. Accordingly, the environment of the luciferin-binding site of PxGR luciferase resembles ethyl acetate, whereas the environment of the luciferin-binding site of PxRE luciferase resembles that of DMSO. A more polar active site environment for the red-emitting luciferase, as suggested by these results, is in agreement with the theory of solvent and orientation polarizability effects on emission spectra $(15,16)$. However, this conclusion should be made with caution, because the environment of the luciferin-binding site when binding these probes could be different from the environment experienced by excited oxyluciferin during the emission step.

Luciferin-Binding Sites of Phrixotrix LuciferasesAccording to Branchini et al. (13) and Ugarova (14), the luciferin-binding site in $P$. pyralis firefly luciferase involves residues R218, 244HHGF247, 315GG316, 340YGLTE344, A348, and I351. In the Phrixotrix luciferases, these positions correspond to R215, 241HHAF245, 312GGA(S)314, 337YGLTE341, A345, and L348, respectively (29). Most of these residues are invariant, with the exception of the conservative substitution of G243 found in the $\mathrm{pH}$-sensitive luciferases with alanine in the $\mathrm{pH}$-insensitive luciferases, a substitution that was previously observed to result in a red shift in the bioluminescence spectrum of the $\mathrm{pH}$-insensitive luciferases (32). In agreement with the experimental data on fluorescence spectra of TNS, the active site model of PxGR luciferase, based on L. cruciata luciferase threedimensional structure complexed with oxyluciferin, is more hydrophobic than that of PxRE luciferase. The substitutions C311T, I334L, I348L and C344S, which are located in the benzothiazolyl side of the luciferin-binding site (Fig. 4), account for the differences in the hydrophobicity between PxGR and PxRE luciferases. Besides being slightly less hydrophobic, the benzothiazolyl binding site of PxRE luciferase is also slightly tighter than that of PxGR luciferase, which could explain the lower $k_{\mathrm{M}}$ value for luciferin. Finally, whereas substitution of the invariant residues $\mathrm{H} 245$ and T343 causes a dramatic red shift in the bioluminescence spectra of firefly luciferases, the corresponding substitution H242A had no effect on the spectra of the railroad worm luciferases, and the T340A substitution resulted in only a slight red shift in the bioluminescence spectrum of PxGR luciferase (Table 3). The only invariant residue within the presumed luciferin-binding site whose substitution had a dramatic effect on the bioluminescence spectra of railroad worm luciferases was R215. Its substitution by serine resulted in a $35 \mathrm{~nm}$ red shift in PxGR luciferase, but had no effect on PxRE luciferase (Table 3) (30). According to the models, this residue is located at 6 and $6.5 \AA$ from the oxyluciferin phenolate of PxGR and PxRE luciferases, respectively. The weak effects observed when most of these invariant residues were substituted in PxGR luciferase suggest that there could be compensating interactions keeping an active-site structure favourable for green light emission. On the other hand, the absolute lack of effect in PxRE luciferase suggests that most of these residues could be misaligned or interact to a lesser degree with excited oxyluciferin in the red-emitting luciferase.

The Guanidine Effect-Other evidence that the luciferinbinding site is different in PxRE luciferase comes from the previous observation that guanidinium ion induces a $20 \mathrm{~nm}$ blue shift in the bioluminescence spectrum of PxRE luciferase (30) (Fig. 5). This effect is very specific, and previous experiments using urea, $\mathrm{KCl}$, and $\mathrm{NaCl}$ ruled out 


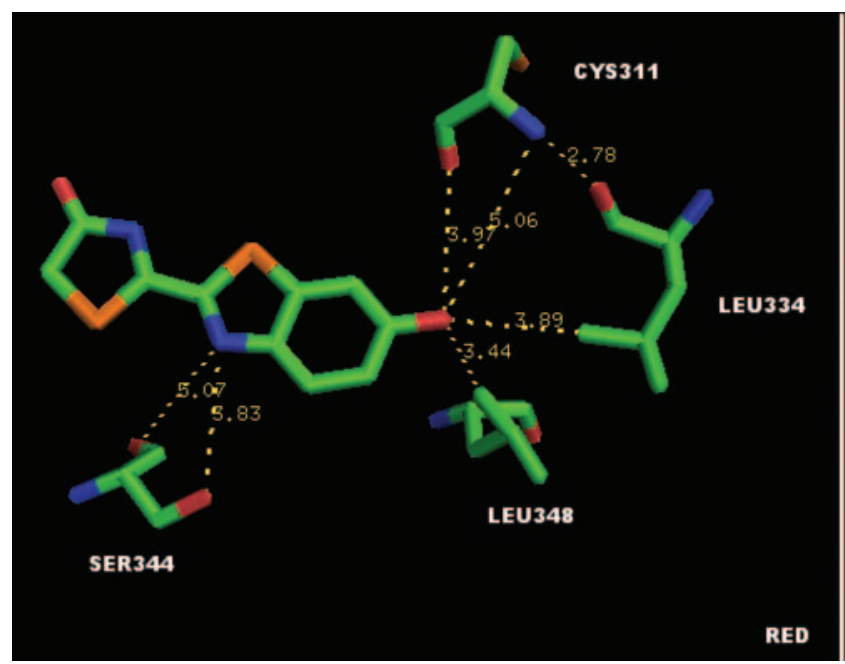

Fig. 4. Three-dimensional models of Phrixotrix railroad worm luciferases based on Luciola cruciata luciferase complexed with oxyluciferin and AMP, showing residues

Table 3. Effects of site-directed mutagenesis of some Phrixotrix railroad worm luciferase luciferin-binding-site residues

\begin{tabular}{lcc}
\hline \begin{tabular}{l} 
Luciferase/mutants $_{\text {[Ppy luciferase] }}^{\mathrm{a}}$ \\
\cline { 2 - 3 }
\end{tabular} & \multicolumn{2}{c}{$\lambda_{\max }(\mathrm{nm})$} \\
\hline Wild-type & 546 & $\mathrm{PxRE}^{\mathrm{c}}$ \\
R215S [R218] & 585 & 623 \\
H242A [H245] & 548 & 615 \\
A243G [G246] & 552 & 619 \\
T340A [343] & 555 & 621 \\
\hline
\end{tabular}

${ }^{a}$ Respective position in Photinus pyralis luciferase. ${ }^{\mathrm{b}} \mathrm{PxGR}$ : Phrixotrix viviani green emitting luciferase. ${ }^{\mathrm{c}} \mathrm{PxRE}$ : Phrixotrix hirtus red emitting luciferase.

non-specific cation or denaturant effects. The absence of the guanidine effect on the fluorescence spectra of both tryptophan and TNS also suggests that the guanidine-induced blue shift in PxRE luciferase does not result from conformational changes. Previously we suggested that guanidine simulates a missing or displaced functional arginine residue in the red-emitting luciferase (30). Such residue could be the invariant Arg215, since its substitution had effect only in PxGR luciferase but not in PxRE luciferase (Table 3) and guanidine was able to shift again the PxGR R215S mutant spectrum to shorter wavelengths (30). Alternatively, guanidine could stabilize the enol form of excited oxyluciferin, which according to recent studies could be the orange light emitter (43).

Relationship between Active-Site Properties and Bioluminescence Colours-According to the former proposed mechanisms for bioluminescence color determination, red bioluminescence is consistent with: (I) polar environments $(15,16)$, (II) lack of specific interactions (25) and (III) with a more relaxed active site (26). Recently, however, Branchini and coworkers suggested that luciferase modulates emission color by controlling the resonancebased charge delocalization of the anionic keto form of excited oxyluciferin (28). Theoretical studies indicated that the degree of polarization of oxyluciferin phenolate/ keto-enolate groups, under influence of the active site

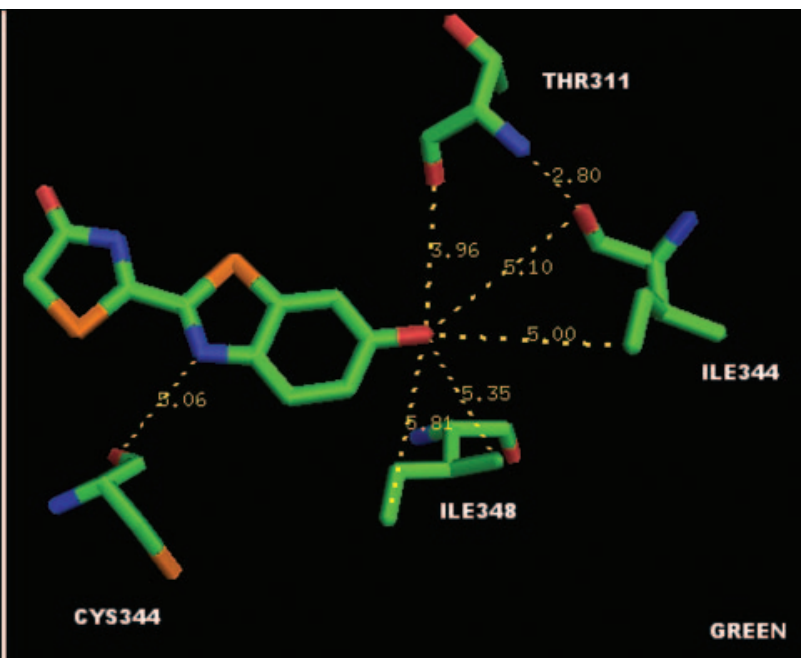

within $6 \AA ̊$ distance from oxyluciferin. (Red) PxRE luciferase (green) PxGRluciferase.

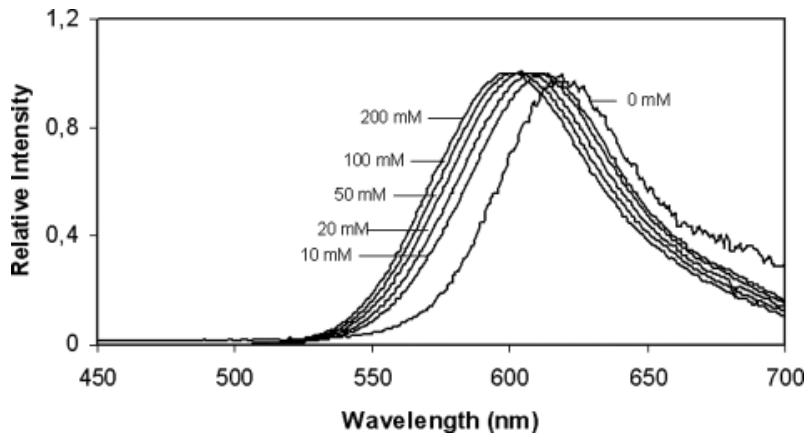

Fig. 5. Effect of guanidine sulfate on the bioluminescence spectrum of purified PxRE railroad worm luciferase.

residues, could be determinant of bioluminescence colors (27). Ugarova and coworkers showed that the bioluminescence spectra of fireflies is a superposition of the spectra of three emitting species: keto (red), enol (orange) and enolate (green) (43). Finally, our recent studies with luciferyl-adenylate chemiluminescence in presence of bovine serum albumin, neutral detergents and solvents, suggest that red chemiluminescence requires less structured environments than green chemiluminescence (44). However, according to these studies, efficient red chemiluminescence requires aprotic environments (44).

The TNS fluorescence spectra agree with a polar and aprotic environment in PxRE luciferase. Furthermore, the lack of effect of some luciferin-binding-site residues substitutions on the emission spectrum of PxRE luciferase agrees with a less structured environment in which some of these residues could be displaced from oxyluciferin during the emitting step. However, the higher affinity of PxRE luciferase for luciferin is apparently inconsistent with a lower degree of active-site interactions and with a more relaxed active site, as required by McCapra et al. hypothesis (26). Considering that the benzothiazolyl moietyis tightly buried and stabilized in the binding site, through hydrophobic forces and phenolate interaction with R215, it is possible that at least during the emitting step, the 
thiazolinic moiety could be exposed to a more polar environment in the case of PxRE luciferase and to a more hydrophobic environment in the case of PxGR luciferase. According to the recently proposed theory of polarization of phenolate and keto/enolate groups $(27,28)$, the substitution of R215 would be expected to affect the emission spectrum by increasing the polarization of excited oxyluciferin, if the keto/enolate moiety is not polarized. This is supported by the experimental observation that the substitution of R215 affects the bioluminescence spectrum only in the green emitting railroadworm and firefly luciferases, but not in the red emitting PxRE luciferase. Therefore, these results suggest that distinct bioluminescence colors in Phrixotrix luciferases could be caused by differences located in the thiazolyl portion of the oxyluciferin binding sites, affecting polarity and specific interactions with excited oxyluciferin.

Very recently, when this work was concluded, the three-dimensional structure of $L$. cruciata firefly luciferase and its red mutant S286N complexed with DLSA (5'-O$[N$-(dehydroluciferyl)sulfamoyl]adenosine analog of luciferyl-adenylate and with oxyluciferin were solved (45). The structure shows the proximity of I288 to the benzothyazolyl moiety and phenolate group, creating a rigid and hydrophobic environment favourable for yellowgreen bioluminescence. In the red mutant $\mathrm{S} 286 \mathrm{~N}$, I288 is displaced and the active site assumes a partially open conformation. Although the authors highlight an integral function for I288 in beetle luciferases, the occurrence of valine in both green and red-emitting Phrixotrix luciferases suggest that this position could not be responsible for changes in the active site polarity and bioluminescence colors in these luciferases.

Conclusions-Despite the similarity of their primary sequences, the Phrixotrix luciferases display remarkably different physicochemical properties. The active site of the red-emitting PxRE luciferase displays substrate affinities, catalytic efficiency, and a dielectric constant higher than those of the PxGR luciferase. These results suggest that the benzothiazolyl side of the luciferin-binding site of PxRE luciferase is tighter and more polar than that of PxGR luciferase. Mutagenesis studies suggest that differences in the thiazolyl portion of the luciferin binding site, during the emitting step, could be responsible for differences of bioluminescence colors in these luciferases.

This work was supported by grants from the Fundação de Àmparo à Pesquisa do Estado de São Paulo (FAPESP) and the Japan Society for the Promotion of Science (JSPS). We extend special thanks to Dr Thérèse Wilson (Harvard University) for comments and for discussing this manuscript, and to Professor Luìs Henrique Catalani (Instituto de Quìmica, Universidade de São Paulo) for the use of his spectrofluorometer. We are also indebted to Zildene Correa and to the Laboratòrio Nacional de Luz Sìncrotron (LNLS, Campinas) for assistance with sequencing, to prof. Dr. Jorg Kobarg (LNLS, Campinas) for providing anti-histidine tag antibodies and to Mr Anderson Rodrigues (UNESP) for his technical assistance.

\section{REFERENCES}

1. Roda, A., Pasini, P., Michelini, E., and Guardigli, M. (2004) Biotechnological applications of bioluminescence and chemiluminescence. Trends Biotechnol. 22, 296-303
2. De Wet, J.R., Wood, K.V., Helinsky, D.R., and DeLuca, M. (1985) Cloning of firefly luciferase cDNA and expression of active luciferase in Escherichia coli. Proc. Natl. Acad. Sci. USA 82, 7870-7873

3. Tatsumi, H., Masuda, T., Kajiyama, N., and Nakano, E. (1989) Luciferase cDNA from Japanese firefly Luciola cruciata: cloning, structure and expression in E. coli. J. Biolum. Chemilum. 3, 75-78

4. Tatsumi, H., Kajiyama, N., and Nakano, E. (1992) Molecular cloning and expression in $E$. coli of a cDNA encoding luciferase of a firefly Luciola lateralis. Biochem. Biophys. Acta 1131, 161-165

5. Devine, J.H., Kutuzova, G.D., Green, V.A., Ugarova, N.N., and Baldwin, T.O. (1993) Luciferase from the East European firefly Luciola mingrelica: cloning and nucleotide sequence of cDNA, overexpression in $E$. coli and purification of the enzyme. Biochem. Biophys. Acta 1173, 121-132

6. Ohmiya, Y., Ohba, N., Toh, H., and Tsuji, F.I. (1995) Cloning, expression and sequence analysis of cDNA for the Japanese fireflies, Pyrocoelia miyako and Hotaria parvula. Photochem. Photobiol. 62, 309-313

7. Sala-Newby, G.B., Thomson, C.M., and Campbell, A.K. (1996) Sequence and biochemical similarities between the luciferases of the glow-worm Lampyris noctiluca and the firefly Photinus pyralis. Biochem. J. 313, 761-767

8. Li Y., Buck, L.M., Scaeffer, H.J., and Leach, F.R. (1997) Cloning and sequencing of a cDNA for the firefly luciferase from Photuris pennsilvanica. Biochim. Biophys. Acta 1339, $39-52$

9. Lee, K.S., Park, H.J., Bae, J.S., Goo, T.W., Kim, I., Sohn, H.D., and Jin, B.R. (2001) Molecular cloning and expression of a cDNA encoding the luciferase from the firefly. Pyrocoelia rufa. J. Biotechnol. 92, 9-19

10. McElroy, W.D. and DeLuca, M. (1978) Chemistry of firefly bioluminescence in Bioluminescence in Action (Herring, P., ed.) pp. 109-127, Academic Press, New York

11. Ugarova, N.N. (1989) Luciferase of Luciola mingrelica fireflies: kinetics and regulation mechanism. J. Biolum. Chemilum. 4, 406-418

12. Conti, E., Franks, N.P., and Brick, P. (1996) Crystal structure of firefly luciferase throws light on a superfamily of adenylateforming enzymes. Structure 4, 287-298

13. Branchini, B.R., Magyar, R.A., Murtishaw, M.H., Anderson, S.M., and Zimmer, M. (1998) Site-directed mutagenesis of Histidine 245 in firefly luciferase: a proposed model of the active site. Biochemistry 37, 15311-15319

14. Sandalova, T.P. and Ugarova, N.N. (1999) Model of the active site of firefly luciferase. Biochemistry (Moscow) 64, 962-967

15. DeLuca, M. (1969) Hydrophobic nature of the active site of firefly luciferase. Biochemistry 8, 160-166

16. Ugarova, N.N. and Brovko, L.Y. (2002) Protein structure and bioluminescence spectra for firefly bioluminescence. Luminescence 17, 321-330

17. Wood, K.W., Lam, Y.A., Seliger, H.H., and, W.D. McElroy (1989) Complementary DNA coding click beetle luciferases can elicit bioluminescence of different colors. Science 244, 700-702

18. Viviani, V.R., Silva, A.C.R., Perez, G.L.O., Santelli, R.V., Bechara, E.J.H., and Reinach, F.C. (1999) Cloning and molecular characterization of the cDNA for the Brazilian larval click-beetle Pyrearinus termitilluminans luciferase. Photochem. Photobiol. 70, 254-260

19. Wood, K.V. (1990) Luc genes: introduction of colors into bioluminescence assays. J. Biolum. Chemilum. 5, 107-114

20. Kajiyama, N. and Nakano, E. (1991) Isolation and characterization of mutants of firefly luciferase which produce different colors of light. Protein Eng. 4, 691-693

21. Ohmiya, Y., Hirano, T., and Ohashi, T.M. (1996) The structural origin of the color differences in the bioluminescence of firefly luciferase. FEBS Lett. 384, 83-86 
22. Mamaev, S.V., Laikhter, A.L., Arslan, T., and Hecht, S.M. (1996) Firefly luciferase: Alteration of the color of emitted light resulting from substitutions at position 286. J. Am. Chem. Soc. 118, 7243-7244

23. Branchini, B.R., Magyar, R.A., Murtishaw, M.H., and Portier, N.C. (2001) The role of active site residue arginine 218 in firefly bioluminescence. Biochemistry 40, 2410-2418

24. Branchini, B.R., Southworth, T.L., Murtishaw, M.H., Boije, H., and Fleet, S.E. (2003) A mutagenesis study of the putative luciferin binding site residues of firefly luciferase. Biochemistry 42, 10429-10436

25. White, E.H. and Branchini, B. (1975) Modification of firefly luciferase with a luciferin analog. A red light producing enzyme. J. Am Chem. Soc. 97, 1243-1245

26. McCapra, F., Gilfoyle, D.J., Young, D.W., Church, N.J., and Spencer, P. (1994) The chemical origin of color differences in beetle bioluminescence in (Campbell, A.K., Kricka, L.J., and Stanley, P.E., eds.) pp. 387-391, Wiley \& Sons, Chichester

27. Orlova, G., Goddard, J.D., and Brovko, L.Y. (2003) Theoretical study of the amazing firefly bioluminescence: the formation and structure of the light emitters. JACS 125, 6962-6971

28. Branchini, B.R., Southworth, T.L., Magyar, R.A., Gonzalez, S.A., Ruggiero, M.C., and Stroh, J.G. (2004) An alternative mechanism of bioluminescence color determination in firefly luciferase. Biochemistry 43, 7255-7262

29. Viviani, V.R., Bechara, E.J.H., and Ohmiya, Y. (1999) Cloning, sequence analysis, and expression of active Phrixothrix railroad-worms luciferases: relationship between bioluminescence spectra and primary structures. Biochemistry 38, 8271-8279

30. Viviani, V.R. and Ohmiya, Y. (2000) Bioluminescence color determinants of Phrixothrix railroad-worm luciferases: chimeric luciferases, site-directed mutagenesis of Arg 215 and guanidine effect. Photochem. Photobiol. 72, 267-271

31. Viviani, V.R., Uchida, A., Suenaga, N., Ryufuku, M., and Ohmiya, Y. (2001) T226 is a key residue for bioluminescence spectra determination in beetle luciferases. Biochem. Biophys. Res. Commun. 280, 1286-1291

32. Viviani, V.R., Uchida, A., Viviani, W., and Ohmiya, Y. (2002) The influence of Ala243(Gly247), Arg215 and Thr226(Asn230) on the bioluminescence spectra and $\mathrm{pH}$-sensitivity of railroad worm, click beetle and firefly luciferases. Photochem. Photobiol. 76, 538-544

33. Viviani, V.R., Silva Neto, A.J., and Ohmiya, Y. (2004) The influence of the region between residues 220 and 344 and beyond in Phrixotrix railroad worm luciferases green and red bioluminescence. Protein Eng. Des. Sel. 17, 113-117
34. Venkatesh, B., Arifuzzaman, M., Mori, H., Taguchi, T., and Ohmiya, Y. (2004) GroEL chaperone binding to beetle luciferases and the implications for refolding when co-expressed. Biosci. Biotechnol. Biochem. 68, 2096-2103

35. Venkatesh, B., Arifuzzaman, M., Mori, H., Suzuki, S., Taguchi, T., and Ohmiya, Y. (2005) Use of GFP tags to monitor localization of different luciferases in E. coli. Photochem. Photobiol. Sci. 30, 1-4

36. Nakajima, Y., Kimura, T., Suzuki, C., and Ohmiya, Y. (2004) Improved expression of novel red- and green-emitting luciferases of Phrixotrix railroad worms in mammalian cells. Biosci. Biotechnol. Biochem. 68, 948-951

37. Nakajima, Y., Ikeda, M., Kimura, T., Honma, S., Ohmiya, Y., and Honma, K. (2004) Bidirectional role of orphan nuclear receptor RORa in clock gene transcriptions demonstrated by a novel reporter assay system. FEBS Lett. 565, 122-126

38. Hastings, J.W. and Weber, G. (1965) The quantum flux of isotopic sources. Photochem. Photobiol. 4, 1049-1050

39. Nakamura, M., Maki, S., Amano, Y., Ohkita, Y., Niwa, K., Hirano, T., Ohmiya, Y., and Niwa, H. (2005) Firefly luciferase exhibits bimodal action depending on the luciferin chirality. Biochem. Biophys. Res. Commun. 331, 471-475

40. Gulick, A.M., Starai, V.J., Horswill, A.R., Homick, K.M., and Escalante-Semerena, J.C. (2003) The $1.75 \AA$ crystal structure of acetyl-CoA synthetase bound to adenosine-5'-propylphosphate and coenzyme A. Biochemistry 42, 2866-2873

41. [OLE8] Branchini, B.R., Southworth, T.L., Murtishaw, M.H., Wilkinson, S.R., Khattak, N.F., Rosenberg, J.C., and Zimmer, M. (2005) Mutagenesis evidence that the partial reactions of firefly bioluminescence are catalyzed by different conformations of the luciferase C-terminal domain. Biochemistry 44, $1385-1393$

42. Zako, T., Ayabe, K., Aburatani, T., Kamiya, N., Kitayama, A., Ueda, H., and Nagamune, T. (2003) Luminescence and substrate binding activities of firefly luciferase N-terminal domain. Biochem. Biophys. Acta 1649, 183-189

43. Ugarova, N.N., Maloshenok, L.G., Uporow, I.V., and Kosharov, M.I. (2005) Bioluminescence spectra of native and mutant firefly luciferases as a function of $\mathrm{pH}$. Biochemistry (Moscow) 70, 1262-1267

44. Viviani, V.R. and Ohmiya, Y. (2006) Bovine serum albumin displays luciferase-like activity in presence of luciferyladenylate: insights on the origin of protoluciferase activity and bioluminescence colors. Luminescence (in press)

45. Nakatsu, T., Ichiyama, S., Hiratake, J., Saldanha, A., Kobashi, N., Sakata, K., and Kato, H. (2006) Structural basis for the spectral difference in luciferase bioluminescence. Nature 440, 372-376 